\title{
GP prescribing of nicotine replacement and bupropion to aid smoking cessation in England and Wales
}

Number of pages: 15

Number of words: 2,271

Andy McEwen, MSc, RMN (Senior Research Nurse) ${ }^{1}$, Robert West, PhD (Professor of Health Psychology) ${ }^{1} \&$ Lesley Owen, PhD (Special Advisor: Smoking) ${ }^{2}$

${ }^{1}$ Health Behaviour Unit (Cancer Research UK), University College London, 2-16 Torrington Place, London, WC1E 6BT

${ }^{2}$ Health Development Agency, Holborn Gate, 330 High Holborn, London, WC1V 7BA

Correspondence to: Andy McEwen, Health Behaviour Unit (Cancer Research UK), University College London, 2-16 Torrington Place, London, WC1E 6BT Tel: 02076796495 Fax:02078132848_andy.mcewen@ucl.ac.uk

Statement of competing interests: Andy McEwen and Robert West have both received research and travel funding from, and undertaken consultancy for, manufacturers of nicotine replacement products.

\section{GP prescribing of nicotine replacement and bupropion}




\section{to aid smoking cessation in England and Wales}

Andy MEwen, Robert West \& Lesley Owen

\section{ABSTRACT}

Aims: Prescribing nicotine replacement therapy (NRT) or bupropion for smoking cessation is of considerable importance to public health but little is known about prescribing practices. This paper examines GPs' prescribing patterns in Britain where these drugs are reimbursed. It also explores what underlies differences between them in prescribing behaviour. The results have implications for other healthcare systems considering introducing reimbursement.

Design, participants and setting: Postal survey conducted in 2002 of a random sample of 1088 GPs in England and Wales of whom 642 (59\%) responded.

Measures: Number of requests GPs reported having received from patients for NRT and bupropion over the past month, the number of prescriptions they reported issuing and ratings of attitudes to these medications.

Findings: GPs reported receiving an average of 4.3 requests for NRT and 1.9 for bupropion in the previous month. They reported issuing 3.5 prescriptions for NRT and 1.2 for bupropion. Almost all GPs accepted that NRT (95\%) and bupropion (97\%) should be reimbursable on National Health Service (NHS) prescription. However, a significant minority of those who received requests for prescriptions did not issue any ( $8 \%$ for NRT and $26 \%$ for bupropion). This was related to whether they thought these products should be available on NHS prescription for both NRT (OR [odds ratio] for 
any prescribing versus none $=0.55, \mathrm{p}<.03)$ and bupropion $(\mathrm{OR}=0.66, \mathrm{p}<.05)$ which in turn was related to beliefs about whether smokers should have to pay for treatment themselves, the cost-effectiveness of NRT/bupropion and the low priority they would give NRT/bupropion in the drug budget. For bupropion, concern about side effects independently predicted not prescribing $(\mathrm{OR}=1.46, \mathrm{p}<.03)$.

Conclusion: In the British healthcare system which has a well-established system for technology assessment and clear professionally endorsed guidelines, a significant minority of GPs decline all patient requests for stop smoking medicines and this appears to relate to broad attitudes to smoking cessation including value judgements about who should bear the cost.

Key words: nicotine replacement therapy (NRT), bupropion, general practitioners (GPs), smoking cessation 


\section{Introduction}

Stopping smoking improves health and reduces the risk of premature death [1], but is made particularly difficult by dependence on nicotine [2]. Both NRT [3] and bupropion $[4,5]$ are effective aids to smoking cessation and are now available on National Health Service (NHS) prescription in the UK (making them reimbursable). The National Institute of Clinical Excellence (NICE) has concluded that they are among the most cost-effective life saving interventions available to the health care system today [6]. National smoking cessation guidelines recommend that GPs should provide accurate information on NRT and bupropion and prescribe these drugs to smokers motivated to stop and seeking help to do so [7]. Following the availability of NRT and bupropion on NHS prescription, since May 2001 and July 2000 respectively, the drug budgets of GPs were increased, although this money was added to the general medicines budget and not restricted to pharmacotherapy for smoking cessation. The general medicines budget is money allocated annually by the government to general practices to cover the estimated cost of all medicines; these finite resources do not commonly result in patients being denied vital medicines, but GPs may have to prioritise their prescribing of non-vital medications.

The extent of demand for these medications and GP prescribing patterns are not known. Neither is it known to what extent GPs believe that it is appropriate for these medications to be part of their treatment armamentarium. We conducted research on this latter issue in 2000 and found only limited support for NRT being placed on NHS prescription [8]. However, the situation has changed substantially since then with updated national smoking cessation guidelines and the medications having actually been placed on the list of reimbursable medications. The issue is of considerable importance both because of the potential for these medications to save many 
thousands of lives and because they may still occupy something of a grey area in thinking about what should be considered a legitimate use of NHS funds. The results should be of interest to other health systems that are considering or will consider reimbursing smoking cessation medications.

\section{Methods}

A postal survey was conducted of a random sample of 1088 GPs in England and Wales conducted between January and March 2002. There were 466 responses to the initial mailing; a second mailing was sent to the non-responders four weeks later, yielding a further 176 responses. In 15 cases the questionnaire was returned uncompleted because the GP had moved away or was no longer practising. The 642 respondents represented an effective response rate of 59\%. The questionnaire was accompanied by a covering letter to the GP and a pre-paid envelope in which to return it; no incentive was given to GPs to complete the survey. In terms of gender, age and, type and location of practice this sample closely resembled national distributions [9, 10]. Sixty-four percent of respondents were male and $10 \%$ worked in single-handed practices. The age distribution was as follows: $<30=1 \%, 30-39=26 \%, 40-49=42 \%$, $50-59=27 \%$ and $>60=5 \%$.

GPs were asked to recall the number of requests they had received from patients for NRT and bupropion over the past month, and the number of prescriptions they had issued. They were further asked whether they thought bupropion and NRT should be available on NHS prescription; and whether they thought these medications should only be available if accompanied by behavioural support or counselling. The responses to these statements were recoded to either "yes" or "no" and "don't know". A number of 
attitude statements relating to smoking cessation and to NRT and Zyban were also presented to GPs requesting that they respond on a five-point Likert scale: strongly disagree, disagree, neutral, agree and strongly agree; although for reporting purposes they have been collapsed to disagree, neutral and agree (see Table 1).

\section{Results}

The mean number of requests made to GPs for NRT in the previous month was 4.3 $($ median $=3$, range: $0-80, \mathrm{SD}[$ standard deviation] $=5.82)$. The mean number of prescriptions issued was $3.5(0-40, \mathrm{SD}=4.63)$; this equated to $80 \%$ of requests. Eleven percent $(n=72)$ of GPs reported having not received any requests for NRT in the previous month and $15 \%(\mathrm{n}=99)$ reported not issuing any prescriptions for NRT.

The mean number of requests made to GPs for bupropion in the previous month was 1.9 (median $=1$, range: $0-30, \mathrm{SD}=3.09)$. The mean number of prescriptions issued was $1.2(0-15, \mathrm{SD}=2.07)$; this equated to $59 \%$ of requests. Over one-third $(37 \%, \mathrm{n}=$ 72) of GPs reported having not received any requests for bupropion in the past month; nearly half $(49 \%, \mathrm{n}=317)$ reported not issuing any prescriptions for bupropion during the same period.

Ninety-five percent $(n=540)$ of GPs thought that NRT should be available on NHS prescription; 71\% $(\mathrm{n}=404)$ thought that it should only be available with behavioural support. A similar proportion $(97 \%, \mathrm{n}=550)$ thought that bupropion should be available on NHS prescription with $80 \%(\mathrm{n}=453)$ believing that it should only be available with behavioural support. 
Attitudes to NRT and bupropion were mostly positive (Table 1) although a substantial majority were concerned about the side effects of bupropion.

TABLE 1 NEAR HERE.

Eight percent $(n=46)$ of GPs who received requests for NRT did not issue any prescriptions at all. The demographic characteristics of these GPs were no different from those that did prescribe. When GPs' responses to requests for NRT were coded to 0 (any prescribing) and 1 (no prescribing) a forced entry logistic regression on to all the attitude items showed that GPs who did not issue any prescriptions were less likely to express the view that NRT should be available on the NHS (OR [odds ratio] $=0.55, \mathrm{p}$ $<.03,95 \%$ CI [confidence interval] 0.33 - 0.91). A further forced entry logistic regression of these responses (coded 1= 'should be available', $2=$ 'should not' or 'don't know') with all the attitude items forced into the model simultaneously, showed that this in turn was positively related to whether GPs thought that: smokers should not have to pay for treatment themselves $(\mathrm{OR}=2.43, \mathrm{p}<.001,95 \% \mathrm{CI} 1.83-3.23)$, and negatively related to the view that NRT is not effective enough to make it worth the NHS paying for it $(\mathrm{OR}=0.34, \mathrm{p}<.001,95 \% \mathrm{CI} 0.25-0.48)$ and the low priority they would give NRT in the drug budget $(\mathrm{OR}=0.35, \mathrm{p}<.001,95 \%$ CI $0.26-0.47)$. See Figure 1 .

\section{FIGURE 1 ABOUT HERE.}

Twenty six percent $(n=102)$ of GPs who received a request for bupropion did not issue any prescriptions. As with NRT a forced entry logistic regression with all attitude items entered showed that it was GPs who did not accept that bupropion 
should be available on NHS prescription that were less likely to prescribe $(\mathrm{OR}=0.66$, $\mathrm{p}<.05,95 \%$ CI 0.65 - 1.14); in addition, concern about side effects also played a significant role $(\mathrm{OR}=1.46, \mathrm{P}<.03,95 \%$ CI $1.05-2.02)$. GPs' views on whether bupropion should be available on NHS prescription were, as with NRT, in turn related to whether GPs thought that smokers should not have to pay for treatment themselves $(\mathrm{OR}=2.08, \mathrm{p}<.001,95 \% \mathrm{CI} 1.59-2.73)$, and negatively related to the view that bupropion is not effective enough to make it worth the NHS paying for it $(\mathrm{OR}=0.28, \mathrm{p}$ $<.001,95 \%$ CI $0.20-0.40$ ) and the low priority they would give bupropion in the drug budget $(\mathrm{OR}=0.47, \mathrm{p}<.001,95 \%$ CI $0.36-0.63)$. GPs who did not prescribe bupropion were 3.6 times more likely not to prescribe NRT (Chi-square $=24.7$, Fishers exact $p$ $<.001)$. See Figure 2.

FIGURE 2 ABOUT HERE.

\section{Discussion}

This survey found that GPs reported modest demand for stop-smoking medications, more for NRT than for bupropion. Their prescribing rate was less than the demand, more so for bupropion than NRT. The difference between the demand for NRT and bupropion and its supply was largely due to a minority of GPs who did not respond positively to any requests for prescriptions for these drugs. Not prescribing the stopsmoking medications was linked to the view that they should not be available on NHS prescription, together in the case of bupropion with concern over side effects. It is possible that GPs did not respond positively to all requests for prescriptions because 
the requests came from patients who were non-nicotine dependent or who were contra-indicated. However, very few smokers are contra-indicated for NRT and this would be unlikely to explain why some GPs did not prescribe at all.

It is apparent that even in a healthcare system with strong technical guidance [6], widely disseminated national guidelines endorsed by professional bodies [7] and government support there remains a proportion of GPs who have not accepted that stop-smoking medications are a legitimate item of NHS expenditure and are acting accordingly. Although internationally there is still debate about whether smokers themselves should bear the cost of treatment to help them stop, in the UK the formal mechanism for addressing this issue is the National Institute of Clinical Excellence and this recognised that nicotine dependence is a disorder that merits treatment as would any other disorder. The NHS does not make patients pay for other costeffective treatments, such as statins or anti-hypertensives, however much the patient may have contributed to his or her condition; there is no difference in this philosophy for patients who are nicotine dependent [6]. This suggests a continued need for GP education so that patients wishing to stop smoking are not disadvantaged through being denied access to medications to aid in their quit attempt. In addition, concerns over bupropion's side-effects are leading a number of GPs not to prescribe this medication at all. Such a blanket refusal may not be in the interest of patients. Again, continued education is required to ensure that the advantages and disadvantages of bupropion to patients are assessed in a balanced fashion.

Although a Cochrane review [3] reported that the overall odds ratio for abstinence with NRT compared with placebo was maintained regardless of the intensity of 
additional support, reservations have been voiced as to whether NRT and bupropion may be less effective without any such support [2]. This, together with the product labelling for bupropion, which states that it should be prescribed as an "adjunct to smoking cessation in combination with motivational support" [11], may be responsible for the very high proportion of GPs who believed that NRT and bupropion should only be available with behavioural support. The existence of smoking cessation services to provide this support, which combined with use of pharmacotherapy further boost smokers' chances of stopping smoking [7], may also have been a factor.

This study was limited by the fact that it relied on self-report. However, the figures for prescriptions per GP match very closely the actual number of prescriptions issued at the time the survey was undertaken [12]. The response rate of $60 \%$ leaves scope for sample bias but the demographic characteristics of the sample were similar to the national population of GPs. Moreover, we found no evidence of differences between the GPs who responded using the first questionnaire and those who only responded after a reminder, suggesting that factors that inhibited responding were not related to our measures of interest.

Finally treatment to aid smoking cessation in the UK is in a state of flux and the situation is likely to continue to change. Our survey is the most up-to-date information currently available and will hopefully serve as a benchmark against which to assess subsequent changes that might arise with the introduction of new policies. 


\section{Acknowledgements}

This study was funded by a grant from the Health Development Agency. We thank the general practitioners who responded for their co-operation and are grateful to Martin Jarvis, Sarah Mitchell, Margaret Reuben and Joy Searle for their assistance with this study. 


\section{References}

1. USDHHS. The health consequences of smoking, nicotine addiction. 1988, US Department of Health and Human Services: Washington.

2. Royal College of Physicians, Nicotine Addiction in Britain. 2000, Royal College of Physicians: London.

3. Silagy, C., Lancaster, T., Stead, L., Mant, D. \& Fowler, G., Nicotine replacement therapy for smoking cessation. 2002, Cochrane Database Systematic Review, Issue 4: Oxford.

4. Hurt, R., Sachs, D., Glover, E., Offord, K., Johnston, J., Dale, L., Khayrallah, M., Schroeder, D., Glover, P., Sullivan, C., Croghan, I. \& Sullivan, P. A comparison of sustained-release bupropion and placebo for smoking cessation. N Engl J Med, 1997. 337(17): p. 1195-202.

5. Jorenby, D., Leischow, S., Nides, M., Rennard, S., Johnston, J., Hughes, A., Smith, S., Muramoto, M., Daughton, D., Doan, K., Fiore, M. \& Baker, T. A controlled trial of sustained-release bupropion, a nicotine patch, or both for smoking cessation. N Engl J Med, 1999. 340(9): p. 685-91.

6. National Institute for Clinical Excellence. Guidance on the use of nicotine replacement therapy (NRT) and bupropion for smoking cessation. 2002, National Institute for Clinical Excellence: London.

7. West, R., McNeill, A. and Raw, M. National smoking cessation guidelines for health professionals: an update. Thorax, 2000. 55: p. 987-999.

8. McEwen, A., West, R. \& Owen, L. General Practitioners' views on the provision of nicotine replacement therapy and bupropion. BMC Central Family Practice, 2001. 2(6).

9. Department of Health, Statistics for General Medical Practitioners in England 1991-2001. 2002, Department of Health: London.

10. NHS Workforce Statitics, General and Personal Medical Services, England and Wales 2001. 2001, Department of Health: London.

11. British Medical Association and Royal Pharmaceutical Society of Great Britain, British National Formulary, Number 45. 2003, The Pharmaceutical Press: London.

12. West, R., DiMarino, M., Gitchell, B. \& McNeill, A. The impact of national policy initiatives on use of medicines to aid smoking cessation. Submitted to Tobacco Control. 
Table 1: GPs' responses to statements relating to smoking cessation and to the effectiveness and cost-effectiveness of NRT and bupropion

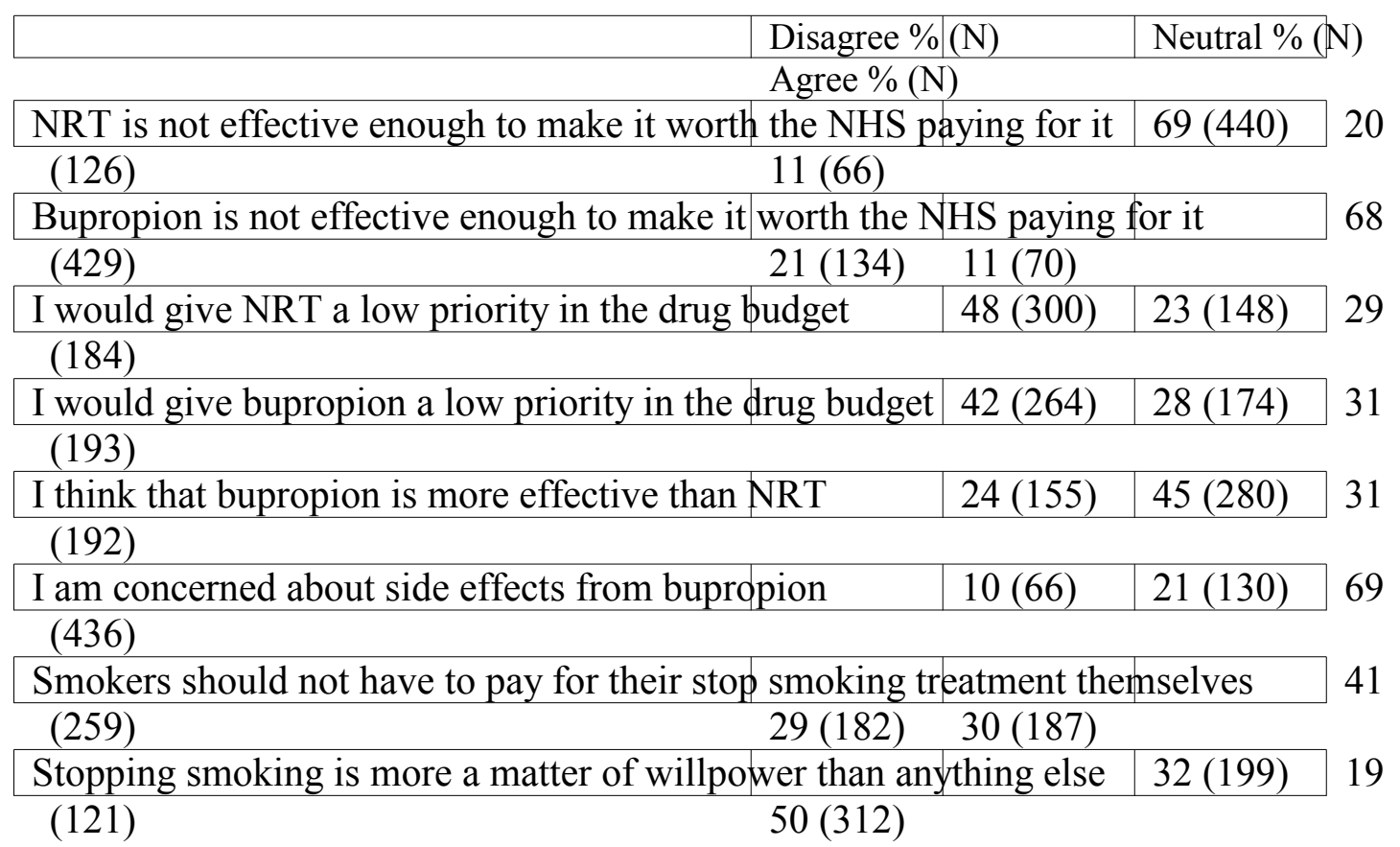

Note: Strongly disagree and disagree, and strongly agree and agree, collapsed in to single responses 
Figure 1: Relationship between whether GPs respond to requests for NRT with a prescription and their attitudes towards NRT

\section{\begin{tabular}{l|l|l} 
Odds Ratio & Odds Ratio
\end{tabular}}

Smokers should not have to pay for their stop smoking treatment themselves 2.43

NRT is not effective enough to make it worth the NHS paying for it

$0.34 \quad$ NRT should be available on NHS prescription

0.55 Did not prescribe NRT

\begin{tabular}{|l|l}
\hline I would give NRT a low priority in the drug budget & 0.35
\end{tabular} 
Figure 2: Relationship between whether GPs respond to requests for bupropion with a prescription and their attitudes towards bupropion

\begin{tabular}{|c|c|c|c|}
\hline & Odds Ratio & \multicolumn{2}{|c|}{ Odds Ratio } \\
\hline \multicolumn{4}{|c|}{ Smokers should not have to pay for their stop smoking treatment themselves } \\
\hline \multicolumn{4}{|l|}{2.08} \\
\hline \multicolumn{4}{|c|}{ Bupropion is not effective enough to make it worth the NHS paying for it } \\
\hline \multirow[t]{2}{*}{0.28} & \multicolumn{3}{|c|}{ Bupropion should be available on NHS prescription } \\
\hline & 0.66 & \multicolumn{2}{|c|}{ Did not prescribe bupropion } \\
\hline \multicolumn{3}{|c|}{ I would give bupropion a low priority in the drug budget } & 0.47 \\
\hline \multicolumn{4}{|c|}{1.46} \\
\hline & & \multicolumn{2}{|c|}{ I am concerned about side effects from bupropic } \\
\hline
\end{tabular}

